\title{
Silicon/soft-carbon nanohybrid material with low expansion for high capacity and long cycle life lithium-ion battery
}

\author{
Naoya Kobayashi ${ }^{\mathrm{a}}$, Yuki Inden ${ }^{\mathrm{b}}$, Morinobu Endo ${ }^{\mathrm{c}, *}$ \\ ${ }^{a}$ Samsung R\&D Institute Japan, 2-7 Sugasawa-cho Tsurumiku, Yokohama 230-0027, \\ Japan \\ ${ }^{b}$ Faculty of Engineering, Shinshu University, 4-17-1 Wakasato, Nagano 380-8553, \\ Japan \\ 'Institute of Carbon Science and Technology, Shinshu University, 4-17-1 Wakasato, \\ Nagano 380-8553, Japan
}

KEYWORDS: lithium-ion battery (LIB), anode, silicon, soft-carbon, hybrid electrode *Corresponding author: endo@endomoribu.shinshu-u.ac.jp

\section{Abstract}

The present study aims at developing a silicon/soft-carbon nanohybrid material for high performance lithium-ion battery (LIB). It is composed of micronized silicon coated with so-called "soft-carbon" dispersed in soft-carbon matrix at nanometer level. This material is characterized with abundant nanosized voids with diameter of ca. $70 \mathrm{~nm}$ and hard bulk skeletal structure. It exhibited a long cycle life of 163 charging and discharging cycles with a large capacity of $850 \mathrm{mAh} / \mathrm{g}$ and retention rate up to $90 \%$ of the initial capacity in a half cell with Li-metal counter electrode. For this new material, the volume expansion ratio was $6.9 \%$ at a 
capacity level of $1100 \mathrm{mAh} / \mathrm{g}$. This electrode capacity is approximately three times larger than that of graphite-based electrode currently used in LIB. Furthermore, this electrode retained $80.9 \%$ of its capacity at 250 cycles in a full cell with a $\mathrm{LiCoO}_{2}$ counter electrode. Addition of 5 wt. \% fluoroethylene carbonate (FEC) to the electrolyte improved the retention up to $81.3 \%$ after 300 cycles. These results demonstrate the usefulness and high possibility of this material as the negative electrode of LIB.

\section{Introduction}

Potential problems associated with natural resources including their depletion and increasing cost and challenges, such as global warming due to the increase in carbon dioxide generated by the combustion of natural resources, have become pronounced in recent years. This has accelerated the expansion of markets for environmentally friendly vehicles such as hybrid electric vehicles, plug-in hybrid electric vehicles, and fuel-cell vehicles. In particular, vehicles are highly anticipated owing to their environmental advantages stemming from their lack of carbon dioxide emissions; however, their actual ranges are still low compared to those of gasoline-powered cars, and the advanced batteries with high energy densities as the power sources of such vehicles are strongly desired to increase their range. In this context, solid-state and metal-air batteries have become increasingly active research and development areas; however, their practical use has not yet been realized.

In this study, we focused on silicon-based electrode materials for lithium-ion battery (LIB); theoretical capacity is approximately 10 times greater than that of graphite electrode of LIB. Hitachi Maxell commercialized SiO negative electrode which is composed of major component of graphite and $\mathrm{SiO}$ additives. Panasonic preliminarily released large capacity battery (4.0 $\mathrm{Ah}$ ) 
with Si-based negative electrode, but not yet commercialized. Thus, no practical application of LIB, taking advantage of large capacity of $\mathrm{Si}$, has been performed. The greatest challenges that obstruct the practical use of silicon-based materials for LIB is their short cycle life that arises from the alloying of silicon and lithium during their charging and discharging processes and the change in their big volume through large expansion and contraction during the repeated desorption of lithium from the alloy. In previous studies, these effects have been well documented to adversely affect the cycle life of LIB. In addition, vehicles require batteries that span the vehicles' expected lifetime of as long as 15 years, which is similar to that of the automobiles based on gasoline internal- combustion engine.

Numerous studies to overcome this lifetime problem have already been conducted, including some reports that a longer battery lifetime can be achieved by lowering the absolute value of the volume change that occurs when silicon and lithium are alloyed; in these cases, longer lifetimes were achieved through the fabrication of thinned silicon electrodes [1-3] and micronization of the silicon $[4,5]$. However, the thin-film method has several shortcomings in terms of production costs that hinder its industrial development; specifically, the required manufacturing equipment is expensive, and its production speed is low compared to that of the method of applying an electrode paste, which is commonly practiced to produce LIB. Furthermore, micronized silicon forms $\mathrm{SiO}_{2}$ via oxidation by oxygen when handled in ordinary atmosphere, which results in increased irreversible capacity during the initial charging of LIB prepared using this method. A large number of studies have been conducted on the conjugation of silicon and graphite [6-21]; however, uniform silicon-graphite composites are difficult to prepare, while the amount of added silicon is difficult to increase.

This study developed a new nanohybrid material, composed of carbon coated micronized silicon dispersed in soft-carbon matrix. It exhibited a long cycle life and suppressed the 
electrode expansion. These behaviors were discussed in connection with abundant nanovoids (diameter of ca. $70 \mathrm{~nm}$ ) and hard bulk skeletal structure.

\section{Experimental method}

2.1 Synthesis of silicon/soft-carbon nanohybrid material

Slurry of silicon / ethanol containing $10 \mathrm{wt} \%$ silicon was prepared using commercially available single-crystalline silicon (manufactured by Kojundo Chemical Laboratory Co., Ltd., $75-\mu \mathrm{m}$ mesh, $99 \%$ purity) and ethanol. The mixture was milled with $\Phi 0.2-\mathrm{mm}$ zirconia balls in an SC10 wet bead mill (Nippon Coke and Engineering Co., Ltd.) for 5 hrs. and then with $\Phi 0.05-\mathrm{mm}$ zirconia balls for $18 \mathrm{hrs}$. [22]. The milled mixture was combined with citric acid and silicon in the ratio of 16.7:83.3 $66.7: 33.3 \mathrm{wt} \%$. It was then transferred to an electric furnace for calcination under flowing argon (flow rate: $500 \mathrm{~cm}^{3} / \mathrm{min}$ ) to obtain carbon-coated silicon granules. The carbonization/calcination procedure consisted of preserving and drying the sample at $60{ }^{\circ} \mathrm{C}$ for $1 \mathrm{~h}$ to remove ethanol, heating it at $500-900{ }^{\circ} \mathrm{C}$ for 4 hours, and then left it to cool to room temperature.

The resulting carbon-coated silicon was mixed with poly-vinyl chloride (PVC, Sigma-Aldrich Corp., Cat. No. 189588), the soft-carbon precursor, in an agate mortar. It was set in an electric furnace for calcination under flowing argon (flow rate: $500 \mathrm{~cm}^{3} / \mathrm{min}$ ). The calcination procedure consisted of heating the sample to a predetermined temperature at a heating rate of $5{ }^{\circ} \mathrm{C} / \mathrm{min}$, holding the sample at $600{ }^{\circ} \mathrm{C}$ for $1 \mathrm{~h}$, and then allowing it to cool naturally. The sample was milled using an agate mortar or a planetary ball mill (P-7 type, Fritsch Co., Ltd.) after calcination. 


\subsection{Characterization of materials}

The cross-sectional structure of the materials was observed using scanning transmission electron microscope (Hitachi High-Technologies Corp.) and HD-2700 field-emission scanning transmission electron microscope (Hitachi High-Technologies Corp.). The carbonization yield of the carbon precursor was determined by TG-DTA analysis (Rigaku TG8120). The structure of the nanohybrid material was determined using Raman microscope (Renishaw) and X-ray diffractometer (Rigaku RINT 2200V/PCSV). The particle size was determined using laser diffraction particle size analyzer (Horiba LA950). The surface area and the pore size distribution were determined by $\mathrm{N}_{2}$-adsorption/desorption analysis at $77 \mathrm{~K}$ (ASAP2020, Micrometrics Co.).

\subsection{Electrochemical characterization}

For the electrochemical measurements of the carbon-coated silicon and the carbon-coated silicon/ soft-carbon nanohybrid material, they were settled on the copper foil according to the following procedures. The carbon-coated silicon, graphite (Hitachi Chemical, Co., MAG) and polyimide (varnish A, Ube Industries, Ltd.) were mixed in a ratio of 40:45:15 wt $\%$, and the viscosity was controlled to a proper value using $N$-methylpyrrolidone. They were coated on a $35-\mu \mathrm{m}$ electro-deposited copper foil by the doctor blade method. The electrode was pressed using a roll press device and the loading level was adjusted to be $2.5 \sim 2.8 \mathrm{mg} / \mathrm{cm}^{2}$. The carbon-coated silicon/soft-carbon nanohybrid material was prepared by mixing the silicon/soft-carbon nanohybrid, carbon black, and polyimide (varnish A) in the ratio of 75:10:15 $\mathrm{wt} \%$ and heating it according to the same manner as noted above. 
For electrochemical test sample was cut into a $\Phi 1.4-\mathrm{cm}$ disc and drying it for $24 \mathrm{~h}$ in a vacuum at $100{ }^{\circ} \mathrm{C}$. It was then transferred to an argon glove box to prepare a 2032-type coin battery by adding $0.1 \mathrm{~cm}^{3}$ of $1 \mathrm{M} \mathrm{LiPF}_{6}$ in an ethylene carbonate (EC)/diethyl carbonate (DEC) $(1: 1$ vol. \%) mixture. The cell was assembled with a polyethylene microporous membrane separator and metal lithium foil or $\mathrm{LiCoO}_{2}$ counter electrode. For the half-cell experiments, the constant current and constant voltage (CCCV) charging and discharging was performed within the range of $0.02(0.10)$ to $1.5 \mathrm{~V}$ at rates of $0.05 \mathrm{C}$ for the first and second cycles, $0.2 \mathrm{C}$ for the third cycle, and $0.5 \mathrm{C}$ for the fourth and subsequent cycles. For the full-cell experiments, the $\mathrm{CCCV}$ charging and discharging was conducted within the range of 4.0 to $3.0 \mathrm{~V}$ at rates of 0.05 $\mathrm{C}$ for the first/second cycles, $0.2 \mathrm{C}$ for the third cycle, and $0.5 \mathrm{C}$ for the fourth and subsequent cycles.

\section{Results and discussion}

\subsection{Carbon-coated silicon fine particle}

The carbon-coated silicon fine particle used in the present investigation was prepared using a wet mill. Silicon precursor with mean particle size of $25 \mu \mathrm{m}$ was milled to fine particles with the mean diameter of ca. $100 \mathrm{~nm}$. The SEM image of silicon fine particle is shown in Fig. 1 (a). The particles have rod-like shape. Several papers claimed that the cycle performance increased with flaky silicon because of the decrease of the absolute value of the electrode expansion [23-25]. Similar effect is expected for the present materials. In addition, the size of the silicon flakes of the present material is in the order of several tenth $\mathrm{nm}$. This is smaller than usually reported value, order of micron $[14,22]$; it should be effective for the depression of the electrode 
expansion. The XRD parameters of pristine and milled silicon particles are shown in Table 1 with the data of BET surface area (Fig. S1). The full width at half maxima (FWHM) increased from $0.129^{\circ}$ to $0.612^{\circ}$ due to the milling. It means that crystallite size changed from $798.7 \mathrm{~nm}$ to $13.7 \mathrm{~nm}$ from the Debye-Scherrer equation. These data suggested a possibility of depressing the expansion in the alloying reaction. The SEM image and EDX of carbon-coated Si/ soft carbon nanohybrid are shown in Fig. 1 (b) and (c), respectively. Si (blue-colored) is dispersed homogeneously in carbon matrix (red-colored). It has been pointed out that a considerable degradation of the cycle performance of the silicon-based electrode occurs during the charging and discharging processes due to the micronization of the particles induced by the volume change in the cycle expansion and shrinkage during alloying and de-alloying with lithium [4]. On the contrary, it has been reported that the cycle performance can be improved by controlling the particle size of the silicon to less than several tenth nm, by which silicon becomes hard to be broken $[4,5]$. However, the micronization of silicon results in large specific surface area as shown in Table 1 and thereby more active sites. The active sites are easily oxidized to silicon oxide in open air, which causes difficulty on the handling because of spontaneous combustion and also increase of the irreversible capacity due to the formation of $\mathrm{SiO}_{2}$. The present investigation tried to coat the surface of the fine particle silicon with carbon to inhibit the oxidation in open air. Citric acid was used for the precursor of carbon, since it is easily soluble in ethanol which was used as the solvent in the milling process. The TG-DTA data of citric acid showed that the carbonization takes place in the temperature range between $200{ }^{\circ} \mathrm{C}$ and $600{ }^{\circ} \mathrm{C}$ (Fig. S2). Based on the residual amount observed in the TG-DTA diagram, the weight ratio of silicon to carbon coated on the surface was estimated. 

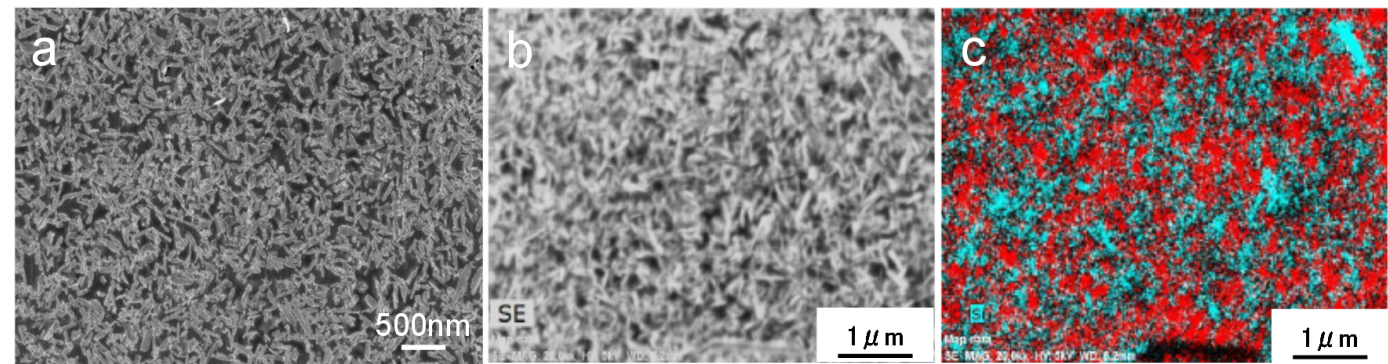

Fig. 1. SEM and EDX of milled Si and carbon-coated Si/ soft carbon nanohybrid: (a), SEM of milled Si; (b), SEM of carbon-coated Si/soft carbon nanohybrid; (c), EDX of carbon-coated Si/soft carbon nanohybrid.

Table 1 XRD parameters and BET surface area of pristine and milled Si.

\begin{tabular}{cccc}
\hline Sample ID & $\begin{array}{c}\text { FWHM } \\
{[\mathrm{deg} .]}\end{array}$ & $\begin{array}{c}\text { Crystallite size } \\
{[\mathrm{nm}]}\end{array}$ & $\begin{array}{c}\text { Bet surface area } \\
{\left[\mathrm{m}^{2} / \mathrm{g}\right]}\end{array}$ \\
\hline Pristine Si & 0.129 & 798.7 & 0.93 \\
\hline Milled Si & 0.612 & 13.7 & 136.57 \\
\hline
\end{tabular}

TEM of the carbon-coated silicon with the $\mathrm{Si} / \mathrm{C}$ weight ratio of 99.3 is shown in Fig. 2. It shows that carbon layer (thickness of ca. $3 \mathrm{~nm}$ ) effectively covered oxide layer (thickness of ca. $2 \mathrm{~nm}$ ) formed on the surface of the rod-like shaped silicon. Because the thickness of the oxide layer is very thin, the carbon coating is effective to protect Si particle from oxidation in air.

Fig. S3 shows the effect of carbonization temperature on the 1st charge and discharge capacity per $1 \mathrm{~g}$ of Si carbon-coated silicon. The maximum discharge capacity is attained at around $600{ }^{\circ} \mathrm{C}$. It reveals that the carbonization of citric acid is insufficient at $500{ }^{\circ} \mathrm{C}$, while the formation of $\mathrm{SiC}$ took place partly at the interface of carbon and silicon at $900{ }^{\circ} \mathrm{C}$. However, XRD measurement showed no SiC phase, showing the extent of SiC phase is very small. The 
SiC phase blocks the diffusion of lithium supplied to the alloying with silicon during charging process. There is a report that even at temperature less than $1000{ }^{\circ} \mathrm{C}$ the formation of $\mathrm{SiC}$ occurred [13] and also the sintering reaction takes place because of mass transfer of the reacting substance at temperature less than the melting point of the material [26]. These reports support above speculation of the formation of $\mathrm{SiC}$ at $900{ }^{\circ} \mathrm{C}$.

Fig. 3 shows the cycle performance of the milled $\mathrm{Si}$ without carbon coating and the carbon-coated milled $\mathrm{Si}$ with the $\mathrm{Si} / \mathrm{C}$ ratio of $99.3 \%$ (CA1-600, heat-treated at $600{ }^{\circ} \mathrm{C}$; CA1-700, heat-treated at $700{ }^{\circ} \mathrm{C}$ ). The milled $\mathrm{Si}$ without carbon coating degraded rapidly in contrast to those with carbon coating. This result clearly showed the carbon coating improves the electrochemical performance. For the advantage of the carbon-coated $\mathrm{Si}$, two possible reasons can be considered. First, the impedance of the electrode due to by-products formed through the reactions with electrolyte during charging process is diminished because of the presence of the surface-coated carbon which prevents the direct contact between $\mathrm{Si}$ and electrolyte. 


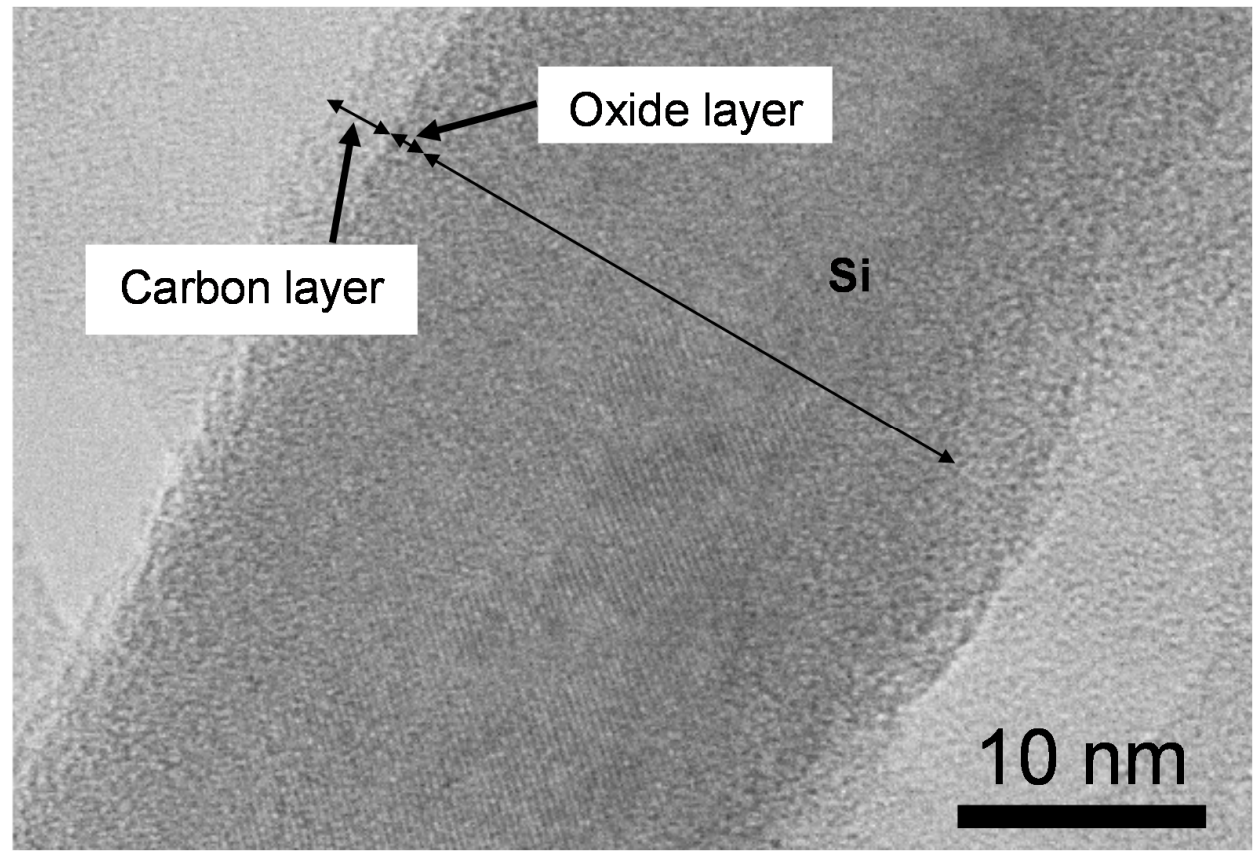

Fig. 2. TEM image of carbon-coated Si.

The other possible reason is that the surface electrically conductive carbon layer assists $\mathrm{Si}$ so as to maintain its conductivity even for the electrode expansion and shrinkage during the charging process. The carbon-coated Si heat-treated at $600{ }^{\circ} \mathrm{C}$ is considered to be the best as the precursor of the subsequent carbon-coated Si / soft-carbon nanohybrid material (Fig. S3). 


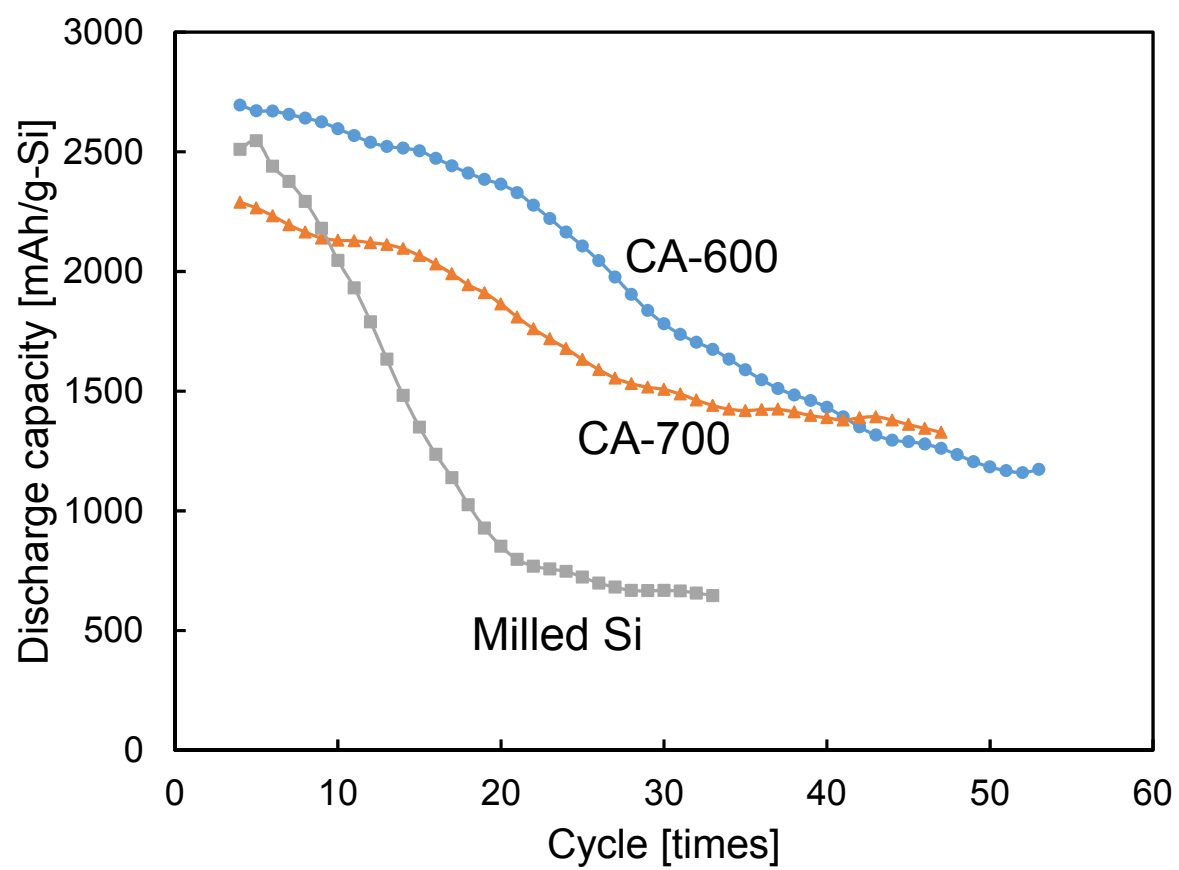

Fig. 3. Cycle performance of milled Si without carbon coating and carbon-coated milled Si with $\mathrm{Si} / \mathrm{C}$ ratio of $99.3 \%$.

\subsection{Carbon-coated $\mathrm{Si} /$ soft-carbon nanohybrid electrode}

As noted in 3.1 the carbon-coating is effective to improve the cycle performance of $\mathrm{Si}$, however it is still insufficient for practical applications. Because soft-carbon contains numerous gaps between its turbostratic carbon units, unlike graphite, and has a harder structure than graphite [27-29], the soft-carbon should be an optimal matrix material for suppressing and assimilating the volume changes of silicon via its conjugation with silicon. Furthermore, the electrode characteristics of soft-carbon differ from those of graphite. The electrode potential gradually approaches toward the potential of lithium during the lithium-ion intercalation process. On the contrary, graphite shows a plateau potential. In the case of graphite and silicon 
composites that have been well studied in the past, silicon is always fully charged against graphite because the alloying potential of silicon with lithium ions is nobler than the lithium-ion intercalation potential of graphite. Such situation facilitates the degradation of Si because silicon rapidly degrades when it is fully charged and discharged [4]. Moreover, the ratio of Si to graphite is fairly small for the nanocomposites of silicon and graphite [30 - 32]. Preparation of nanocomposites of silicon and graphite requires high temperature; high temperature condition tends to generate $\mathrm{SiC}$, which adversely affects battery performance [13]. In contrast, the silicon/soft-carbon nanohybrid materials prepared in the present study have no limitations on the Si content because soft-carbon is synthesized at several hundred degrees, which is less than the formation temperature of $\mathrm{SiC}$. It can suppress the deterioration of silicon due to the full charging and discharging through control of its charge amount in the soft-carbon matrix.

Since PVC was superior as the precursor of soft-carbon among several substances, it was used for preparing composite with the carbon-coated Si. A fixed amount of PVC and Si was mixed (with a ratio which brings about the final $\mathrm{Si} /$ soft-carbon ratio of 62.3:37.7 wt. \%) and heat-treated at $600{ }^{\circ} \mathrm{C}$. The SEM-EDX of the resulting material is shown in Fig. S4, where blue and red spots correspond to Si and carbon, respectively. It shows that the hybrid is composed of Si homogeneously covered with carbon.

The silicon/soft-carbon(SC) nanohybrid materials with ratios, ranging from 57.3/42.7 to 72.3/27.7 wt. \% were prepared and used for fabricating half cells with a lithium-metal counter electrode. The cycle performances of the half cells are shown in Fig. 4, where CCCV charging and discharging condition is $0.5 \mathrm{C}$ and $100 \mathrm{mV}$. Fig. 4 indicates that good cycle performance is seen for the silicon/soft-carbon nanohybrid when the ratio is equal to $62.3 / 37.7 \mathrm{wt}$. \%. It showed longer cycle life (106 cycles with $90 \%$ capacity retention with respect to the initial capacity when cycled at a $0.5 \mathrm{C}$ charge/discharge rate). 


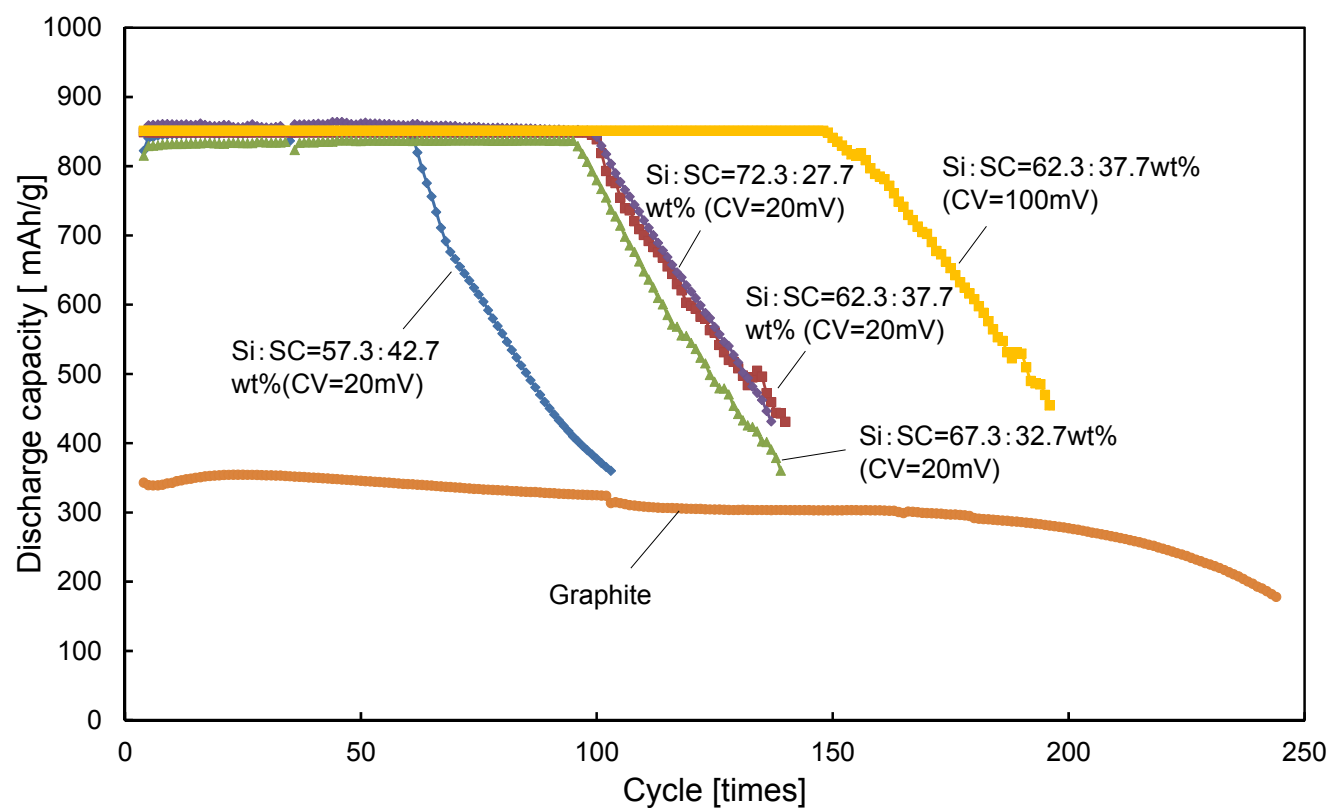

Fig. 4. Influence of the ratio of Si/soft-carbon (SC) nanohybrid on cycle performance of half cell.

SEM image of the cross section of silicon/soft-carbon nanohybrid (62.3/37.7 wt. \%) after charging and discharging cycles is shown in Fig. S5, where SEM image taken before electrochemical test is also shown for comparison. The discharged specimen was extracted from the cell under argon atmosphere in a glove box after 30 times of charging and discharging between 20 and $1500 \mathrm{mV}$; the SEM specimen was in the state of discharged. Comparison of the images of the nanohybrid before and after charging and discharging cycles shows that no significant difference exists between them. The discharged one does not have damages such as cracks and shape change, etc. The present silicon/soft-carbon nanohybrid is able to maintain its structure by absorbing stress due to expansion and contraction of Si during charging and discharging cycles. The carbon layer coated on Si has a role of shock absorber and protector from direct contact between $\mathrm{Si}$ and electrolyte. 
In the Raman spectra shown in Fig. 5, peaks due to silicon appears at approximately 1500 $\mathrm{cm}^{-1}$ for the samples whose silicon/soft-carbon ratio exceeds $67.3 / 32.7 \mathrm{wt}$. \%. It means that soft-carbon does not perfectly covered silicon for these samples. Thus, silicon/soft-carbon ratio of $62.3 / 37.7$ wt. $\%$ is preferable for the homogeneous reaction at the electrode. This electrode can absorb stress due to expansion during alloying reaction with Li because the composite is uniformly conjugated with soft-carbon matrix. It also exhibits good electrical conductivity and thereby a long cycle life. Thus perfect carbon coating on Si is a key factor. To establish coating technique, effect of molecular weight of PVC (precursor of soft carbon matrix) is also be clarified.

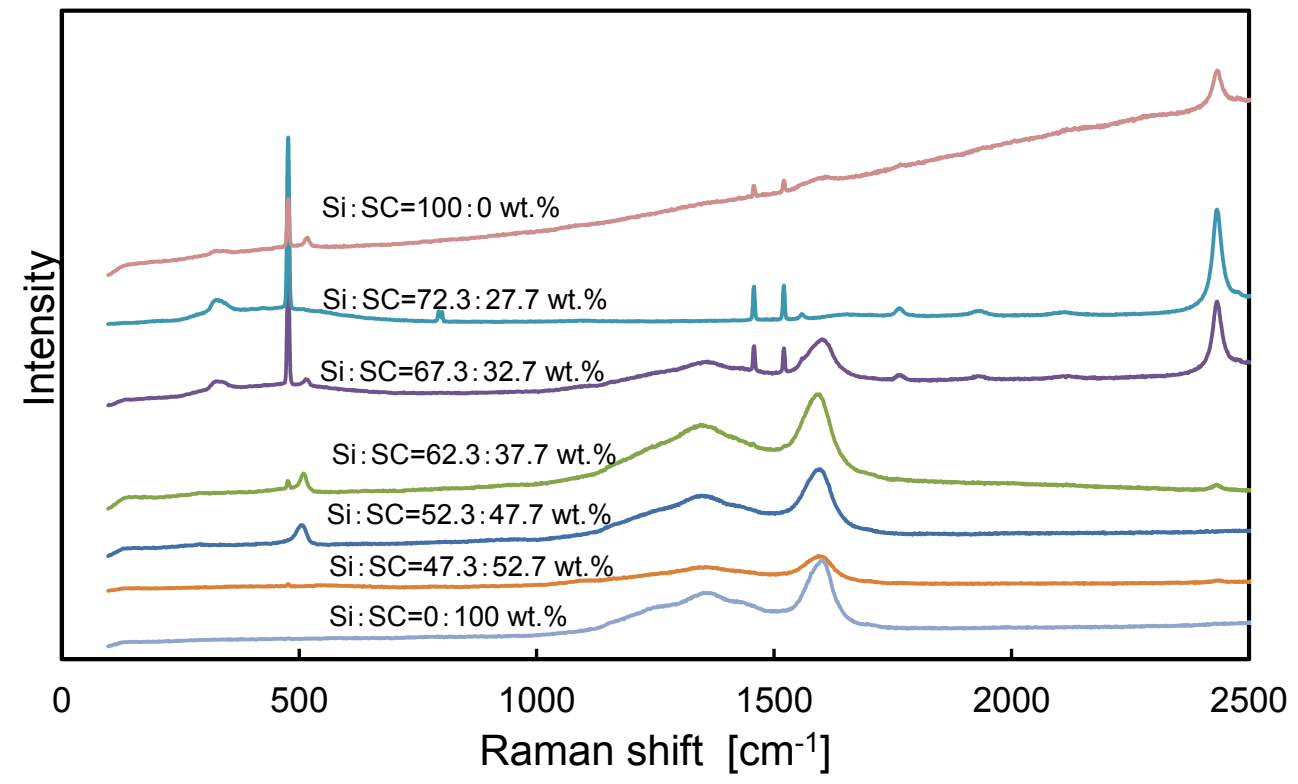

Fig. 5. Raman spectra of Si/soft-carbon nanohybrid combined in various ratios of $\mathrm{Si}$ and hard carbon.

The Si/soft-carbon nanocomposites were prepared from mixture of $\mathrm{Si}$ and PVC with 5 different molecular weight. The preparation conditions are the same as noted above. Raman 
spectra of the resulting nanocomposites are shown in Fig. S6. It clearly indicates that only nanocomposite prepared from PVC with molecular weight of 62000 succeeds complete carbon coating. Fig. S7 is the half-cell cycle performance of the 5 nanocomposites. AC impedance measurements were carried out for SiSC62000 and SiSC80000. Fig. S8 shows the Nyquist plots after the $1^{\text {st }}$ charging. The circle of high frequency region of SiSC62000 is larger than that of SiSC80000, therefore resistance of SiSC62000 is less than that of SiSC80000. It suggests that effective carbon coating of SiSC62000 inhibits the reaction between Si and electrolyte compared with that of SiSC80000. According to the equivalent circuit of the electrode system shown in Fig. S9, film resistance $\left(R_{\text {sei }}\right)$, charge-transfer resistance $\left(R_{\mathrm{ct}}\right)$ and interfacial contact resistance $\left(R_{\mathrm{int}}\right)$ are calculated and summarized in Table S1 (SiSC62000) and Table S2 (SiSC80000). $R_{\text {sei }}$ after $1^{\text {st }}$ charging of SiSC62000 is less than that of SiSC80000. The amount of SEI formed on the electrode is less for SISC62000 than for SiSC80000 as noted above. $R_{\mathrm{ct}}$ of SiSC62000 increased up to 20 cycles and then stabilized until 80 cycles. On the contrary, $R_{\mathrm{ct}}$ of SiSC80000 continues to increase with increasing cycle number. This observation explains the difference of the electrochemical cycle performance of them. SiSC62000 established a good cycle performance because the carbon layer protected Si to react with electrolyte and therefore inhibited SEI formation. On the other hand, SiSC80000 showed degradation with increasing cycle number because carbon coating was not effective and a large number of Si contacted directly with electrolyte.

Fig. S10 shows cyclic voltammogram (CV) of SISC62000 with scanning rate of $0.1 \mathrm{mV} / \mathrm{s}$ and voltage between 20 and $2000 \mathrm{mV}$. CV does not show current corresponding to SEI formation in the region of reduction at about $1 \mathrm{~V}$ for the $1^{\text {st }}$ cycle. This fact agrees well with the fact that $R_{\text {sei }}$ of the $1^{\text {st }}$ cycle is small. The oxidation and reduction peak potential is fairly constant and the shape of CV was stabilized after 3 cycles. 
Fig. S11 shows discharging rate performance of SiSC62000 for half cell. The discharge capacity at $0.2 \mathrm{C}, 0.5 \mathrm{C}, 1 \mathrm{C}$ and $3 \mathrm{C}$ was $1066,1013,1005$ and $976 \mathrm{mAh} / \mathrm{g}$, respectively. Even for rapid discharging such as $3 \mathrm{C}$, the capacity was $91.6 \%$ of that for $0.2 \mathrm{C}$. The discharging voltage was stable with small polarization. The good performance of SiSC62000 is attributed to small load due to micronized Si and high conductivity protected by the existence of soft-carbon.

Silicon exhibits several potential plateaus, depending on the alloying ratio in the alloying reaction with lithium $[33,34]$. The cycle performance at $100 \mathrm{mV}$ for the optimal composition of 62.3/37.7 wt $\%$ showed that its cycle life increased to 163 with increasing CV from 20 to 100 $\mathrm{mV}$ (Fig. 4), which represents a 1.5 -fold increase in cycle life compared to that at a $\mathrm{CV}$ of 20 $\mathrm{mV}$. This reason for improved cycle life at higher cutoff potential is that highly lithiated Si suddenly crystallizes below $50 \mathrm{mV}$ in Li15Si4 crystalline phase. Higher cutoff potential simply prevents crystalline phase formation and LixSi is amorphous during the entire recharge cycle [35]. Suppressing silicon particle expansion is caused by a favorable control of the deep charging (alloying) of silicon in the cycle tests. On the basis of the results obtained for the aforementioned half cell, the usefulness of the nanohybrid material with the composition of 62.3/37.7 wt $\%$ was estimated using the full cell. Using $\mathrm{LiCoO}_{2}$ as the positive electrode, we fabricated and evaluated a coin cell using the same electrolyte as that used in the half cell (1 M $\mathrm{LiPF}_{6}$ in EC/DEC (1:1)); its cycle performances are shown in Fig. 6. The material exhibited a long cycle life: it retained $80.9 \%$ of its capacity after 250 cycles, and long cycle-life performances were achieved even in the full cell wherein $\mathrm{LiCoO}_{2}$, whose lithium content was limited, was used as a positive electrode. Moreover, when $5 \mathrm{wt} \%$ fluoroethylene carbonate (FEC) was added to the electrolyte, the silicon/soft-carbon nanohybrid retained $81.3 \%$ of its initial capacity after 300 cycles. Addition of FEC brought about higher efficiency in the 
charging and discharging cycles. It is due to suppression of the SEI formation by the presence of FEC.

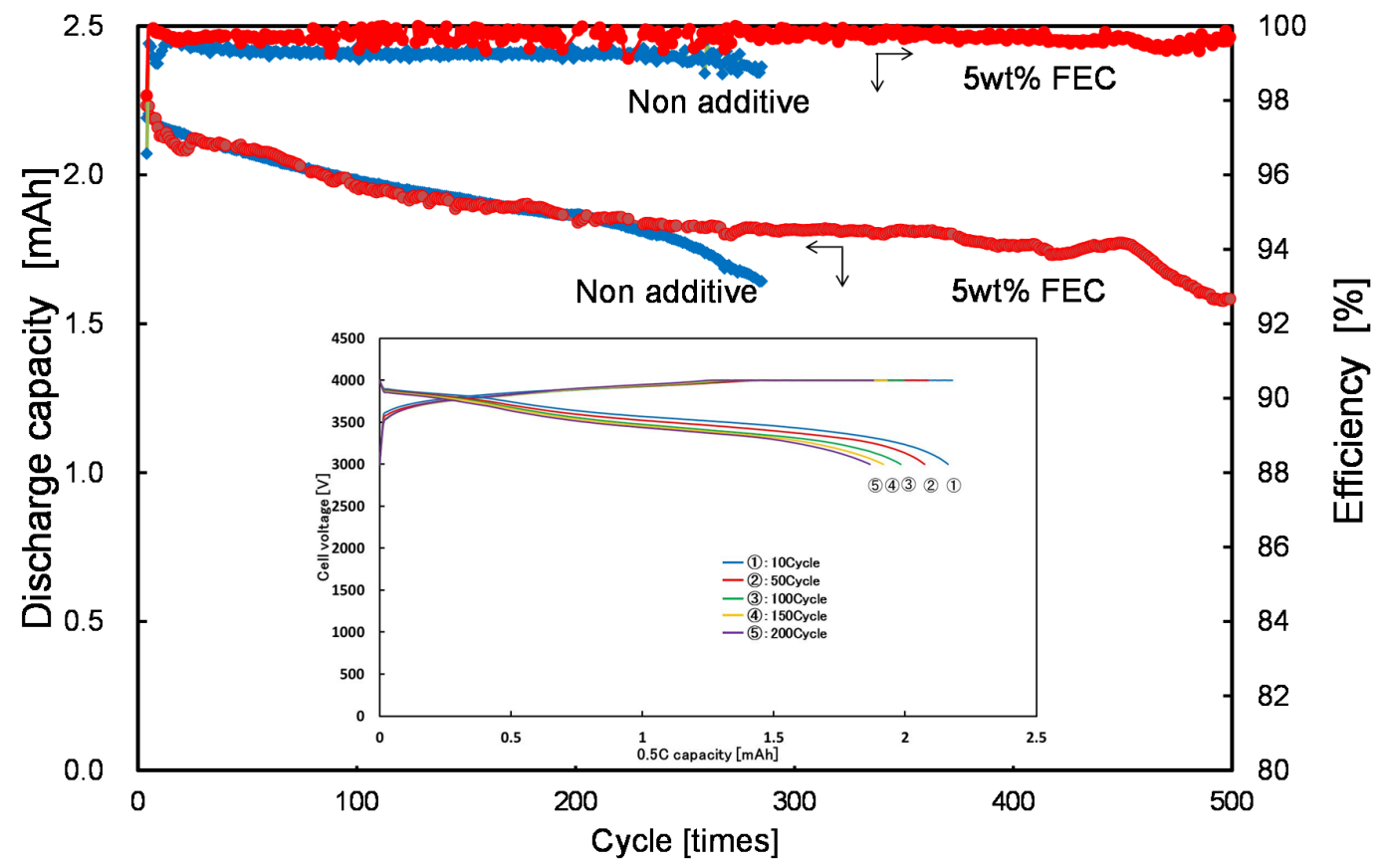

Fig. 6. Cycle performances of $\mathrm{Si} /$ soft-carbon nanohybrid materials $/ \mathrm{LiCoO}_{2}$ batteries.

Electrodes with different density were prepared by pressing the silicon/soft-carbon nanohybrid with a ratio of $62.3 / 37.7 \mathrm{wt} \%$ using a roll press device. These half cells were charged to $1100 \mathrm{mAh} / \mathrm{g}$ at $0.05 \mathrm{C}$. Their thicknesses was measured by removing the electrodes after disassembling the cells in a glove box and the expansion rate of the silicon-based electrodes was calculated on the basis of the difference between their thickness before and after the tests. The results are shown in Table 2. The volume changes of the electrodes were $6.9 \%$ for the electrode with a density of $0.59 \mathrm{~g} / \mathrm{cm}^{3}$ (the degree of expansion: $2 \mu \mathrm{m}$ ), $10.9 \%$ for 0.83 $\mathrm{g} / \mathrm{cm}^{3}$ (the degree of expansion: $5 \mu \mathrm{m}$ ), and $19.2 \%$ for $0.93 \mathrm{~g} / \mathrm{cm}^{3}$ (pressed under the maximum pressure that could be applied with the roll press machine used in this work). In the case of 
low-density electrodes, the expansion force could have been absorbed by air gaps in the electrodes, resulting in a decrease in the electrode gap with increasing electrode density and a greater electrode expansion ratio. The graphite electrode was also evaluated for comparison. Its capacity was $381 \mathrm{mAh} / \mathrm{g}$, which was equivalent to the theoretical capacity of graphite, and their expansion ratio was $20.0 \%$ (the degree of expansion: $14 \mu \mathrm{m}$ ). The silicon/ soft-carbon nanohybrid with an electrode density of $0.93 \mathrm{~g} / \mathrm{cm}^{3}$ exhibits an electrode expansion ratio of $19.2 \%$, being equal to or less than that of graphite. The absolute value of the electrode expansion of the silicon/soft-carbon nanohybrid is lower than that of graphite. Thus, the present electrode exhibits an expansion ratio similar to that of graphite and the capacity of $1100 \mathrm{mAh} / \mathrm{g}$, which is approximately three times greater than that of graphite.

Table 2 Electrode expansion of Si/soft-carbon nanohybrid materials compared with graphite electrode.

\begin{tabular}{cccccc}
\hline Item & $\begin{array}{c}\text { Charge capacity } \\
{[\mathrm{mAh} / \mathrm{g}]}\end{array}$ & $\begin{array}{c}\text { Electrode } \\
\text { density }\left[\mathrm{g} / \mathrm{cm}^{3}\right]\end{array}$ & $\begin{array}{c}\text { Charge capacity } \\
{[\mathrm{mAh} / \mathrm{cm} 3]}\end{array}$ & $\begin{array}{c}\text { Expansion } \\
{[\mu \mathrm{m}]}\end{array}$ & $\begin{array}{c}\text { Expansion rate } \\
{[\%]}\end{array}$ \\
\hline Graphite & 381 & 1.64 & 625 & 14 & 20.0 \\
\hline \multirow{2}{*}{ Si/Soft carbon } & 1100 & 0.59 & 649 & 2 & 6.9 \\
nanohybrid & 1100 & 0.83 & 913 & 5 & 10.9 \\
& 1100 & 0.93 & 1023 & 5 & 19.2 \\
\hline
\end{tabular}

Fig. 7 shows the dependence of the electrode expansion and the total charge capacity on the cycle number during further charge-discharge cycles, where the left ordinate denotes the electrode expansion and right ordinate corresponds to the total charge capacity of the electrode. The total charge capacity involves not only the irreversible capacity which was not able to be discharged in the charge-discharge cycles before the measurement of the electrode expansion but also the charge capacity $(1100 \mathrm{mAh} / \mathrm{g})$ in the measurement of the electrode expansion. Because of the large irreversible capacity in the first charge-discharge cycle and relatively small 
irreversible capacity in the following cycles, the total charge capacity increased very slightly with the cycle number. As seen from Fig. 7, the electrode expansion at fifth cycle increased $1.2 \%$ compared to that at first cycle, while no further increase of the total charge capacity was observed from fifth to tenth cycle, suggesting no additional electrode expansion. The magnitude of the electrode expansion of the silicon/soft-carbon nanohybrid even after tenth charge-discharge cycles is small, therefore this material has a function of preventing the electrode expansion during charging and discharging processes. This behavior brings about the excellent cycle performance of the half cell and the full cell.

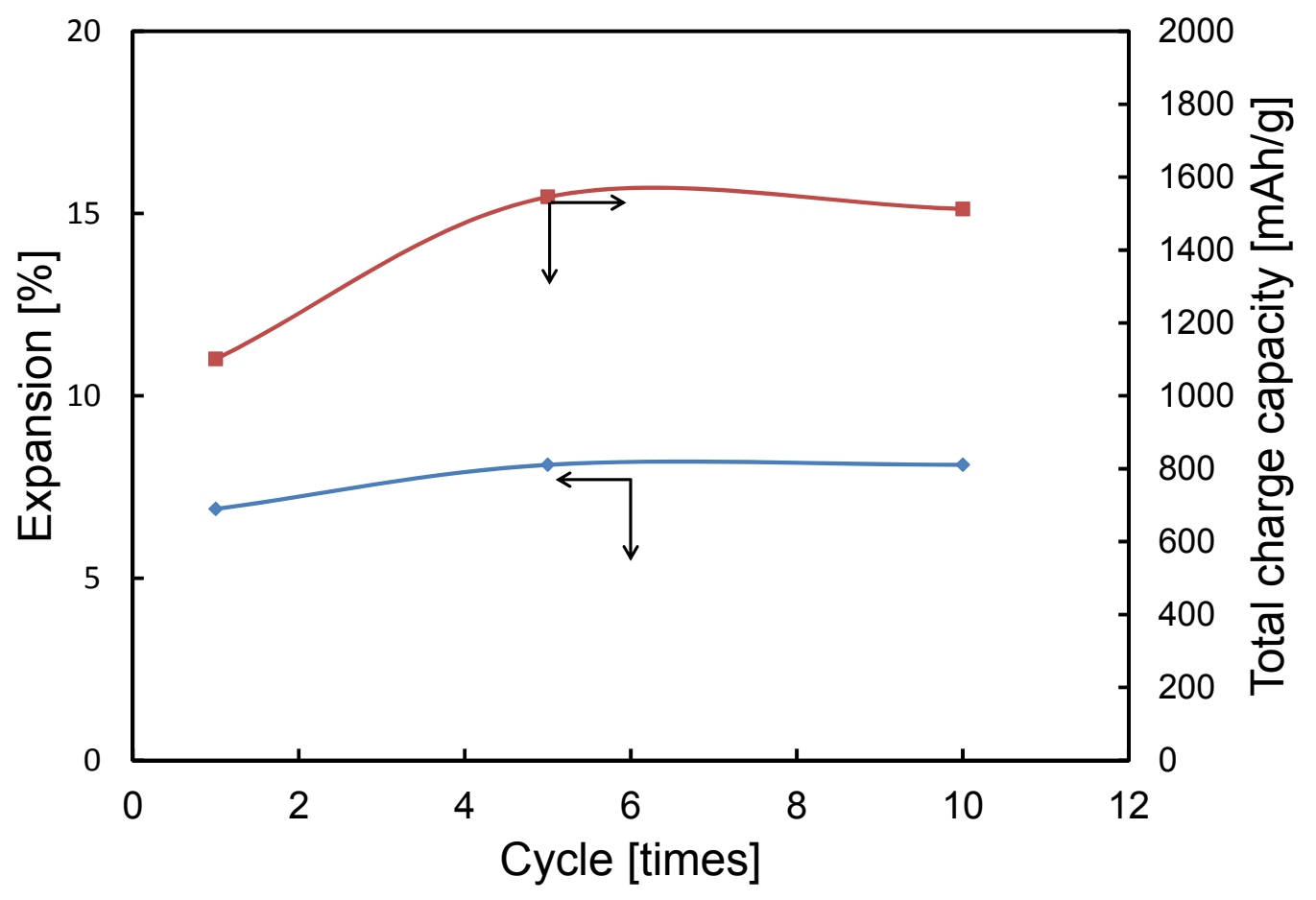

Fig. 7. Electrode expansion and total charge capacity of Si/soft-carbon nanohybrid electrode plotted as a function of cycle number. 
The pore-distribution profiles of soft carbon and silicon/soft-carbon nanohybrid (62.3/37.7 wt. \%) can be seen in Fig. S12. They are quite similar with mesopores (30 40 nm) and macropores $(>50 \mathrm{~nm})$. It means that the pore structure of the soft carbon determines that of nanohybrid. The pores of the nanohybrid have an important role of absorbing the stress due to expansion of Si during electrochemical alloying of $\mathrm{Li}$.

On the basis of the aforementioned results, a structural model of the silicon/soft-carbon nanohybrid material is shown in Fig. 8. Silicon expansion was ameliorated by fabricating a nanohybrid structure consisting of particulate silicon coated with carbon and soft-carbon with voids as the matrix material; this material suppresses air oxidation, which is one of the causes of irreversible capacity of silicon electrodes. As a result, we have successfully developed the silicon-based electrode material for LIB that can achieve both long cycle life and high capacity.
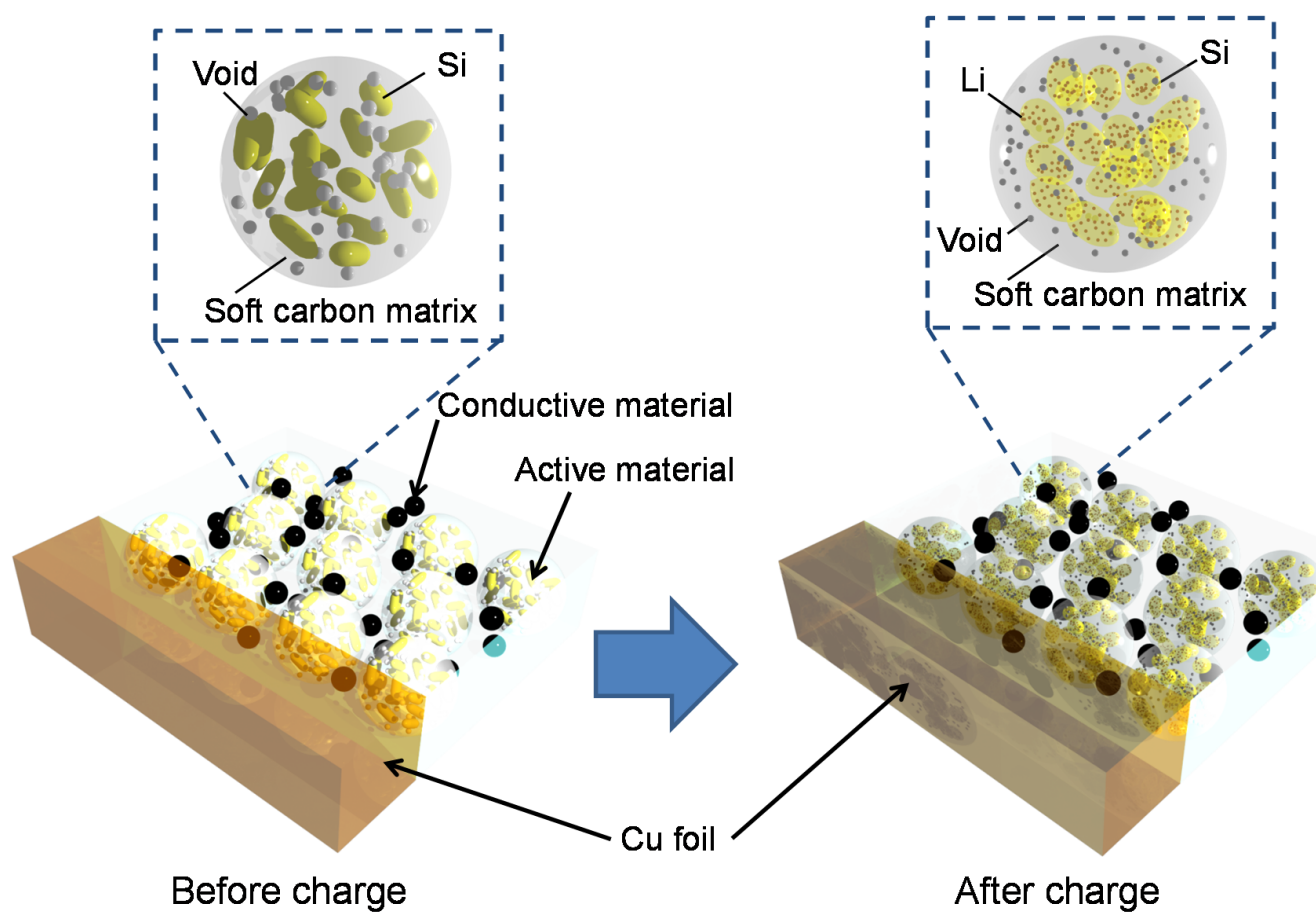

Fig. 8. A structural model of the Si/hard-carbon nanohybrid material. 


\section{Conclusions}

The present study developed silicon/soft-carbon nanohybrid electrode material for the negative electrode of the advanced LIB. The half cell constructed using the material with the silicon/soft-carbon ratio of $62.3 / 37.7 \mathrm{wt} \%(\mathrm{CV}=20 \mathrm{mV})$ exhibited the capacity of $850 \mathrm{mAh} / \mathrm{g}$ for 106 charge-discharge cycles. The cycle life was extended to 163 cycles when the cutoff voltage was increased to $100 \mathrm{mV}$. The full cell composed of the silicon/soft-carbon nanohybrid material with the silicon/soft-carbon ratio of $62.3 / 37.7 \mathrm{wt} \%$ and $\mathrm{LiCoO}_{2}$ counter electrode retained $80.9 \%$ of its capacity after 250 charge-discharge cycles. Addition of 5 wt. \% FEC to electrolyte improved the capacity retention to $81.3 \%$ at 300 charge-discharge cycles. An electrode composed of the silicon/soft-carbon nanohybrid material with a $62.3 / 37.7 \mathrm{wt} \%$ ratio exhibited an electrode expansion rate of $19.2 \%$, even for the electrode with the density of 0.93 $\mathrm{g} / \mathrm{cm}^{3}$ (under the maximum pressurization of the electrode), and the capacity of $1100 \mathrm{mAh} / \mathrm{g}$, which was approximately three times greater than that of graphite electrode. The volume expansion ratio was $6.9 \%$ for the electrode with the density of $0.59 \mathrm{~g} / \mathrm{cm}^{3}$ at a capacity level of $1100 \mathrm{mAh} / \mathrm{g}$. Thus, this material exhibited expansion characteristics equal to or less than that of graphite, which exhibited an electrode expansion rate of $20.0 \%$, thereby confirming its usability.

Consequently, the silicon/soft-carbon nanohybrid material exhibited a practically available long enough cycle life. The capacity was approximately three times larger than that of graphite electrode; electrode expansion rate was comparable to that of graphite currently used. It should be a promising next-generation and high-capacity electrode material for replacing graphite. It will positively affect battery systems for portable electronics as well as for electric vehicles. In the future, this material could be developed into a battery system with greater capacity and 
longer cycle life by combining it with optimized positive electrode materials (e.g., Co- or Ni-, Mn- based materials) and electrolytes.

\section{References}

[1] Tao Hang, Hiroki Nara, Tokihiko Yokoshima, Toshiyuki Momma, Tetsuya Osaka, Journal of Power Sources 222 (2013) 503-509.

[2] Norihiro Shimoi, Yasumitsu Tanaka,Electrochimica Acta 80 (2012) 227-232.

[3] Yongfeng Tong, Zhuang Xu, Chang Liu, Guang'an Zhang, Jun Wang, Z.G. Wu, Journal of Power Sources 247 (2014) 78-83.

[4] Uday Kasavajjula, Chunsheng Wang, A. John Appleby, Journal of Power Sources 163 (2007) 1003-1039.

[5] Shinichiroh Iwamura, Hirotomo Nishihara, Takashi Kyotani, Journal of Power Sources 222 (2013) 400-409.

[6] Nikolay Dimov, Satoshi Kugino, Masaki Yoshio, Electrochimica Acta 48 (2003) 1579-1587.

[7] Nikolay Dimov, Kenji Fukuda, Tatsuo Umeno, Satoshi Kugino, Masaki Yoshio, Journal of Power Sources 114 (2003) 88-95.

[8] Masaki Yoshio, Takaaki Tsumura, Nikolay Dimov, Journal of Power Sources 146 (2005) $10-14$.

[9] Masaki Yoshio, Satoshi Kugino, Nikolay Dimov, Journal of Power Sources 153 (2006) 375-379.

[10] Masaki Yoshio, Takaaki Tsumura, Nikolay Dimov, Journal of Power Sources 163 (200 6) 215-218. 
[11] Nikolay Dimov, Yonggao Xia, Masaki Yoshio, Journal of Power Sources 171 (2007) 886-893.

[12] Q. Si, M. Kawakubo, M. Matsui, T. Horiba, O. Yamamoto, Y. Takeda, N. Seki, N. Imanishi, Journal of Power Sources 248 (2014) 1275-1280.

[13] Y. Liu, Z.Y. Wen, X.Y. Wang, A. Hirano, N. Imanishi, Y. Takeda, Journal of Power Sources 189 (2009) 733-737.

[14] Mingru Su, Zhixing Wang, Huajun Guo, Xinhai Li, Silin Huang, Wei Xiao, Lei Gan, Electrochimica Acta 116 (201) 230-236.

[15]Mingru Su, Zhixing Wang, 24, 6, (2013) 921-925

[16] Caili Zhang, Materials Letters171(2016)63-67

[17] Heng Jiang, Electrochimica Acta 188 (2016) 777-784

[18] Lianlin Deng, Electrochimica Acta 192 (2016) 303-309

[19] Dafang He, Electrochimica Acta 169 (2015) 409-415

[20] Jingbo Chang, Nano Energy (2015) 15, 679-687

[21] Mahmut Dirican, Electrochimica Acta 169 (2015) 52-60

[22] Toshihiro Ishii, Kazuaki Hashimoto, Journal of the Japan Society of Colour Material 84 (2011) 163-168.

[23] Morihiro Saito, Kenta Nakai, Tomoyuki Yamada, Toshio Takenaka, Makoto Hirota, Akika Kamei, Akimasa Tasaka, Minoru Inaba, Journal of Power Sources 196 (2011) 6637-6643.

[24] Morihiro Saito, Kenta Nakai, Tomoyuki Yamada, Toshio Takenaka, Makoto Hirota, Akika Kamei, Akimasa Tasaka, Minoru Inaba, Journal of Power Sources 196 (2011) 6637-6643.

[25] Takashi Okubo, Morihiro Saito, Chihiro Yodoya, Akika Kamei, Masato Hirota, Toshio Takenaka, Toyoki Okumura, Akimasa Tasaka, Minoru, Solid State Ionics 262 (2014) 39-42. 
[26] O. Ertugrul, H.-S. Park, K. Onel, M. Willert-Porada, Powder Technology 253 (2014) 703-709.

[27] Yinghu Liu, J.S. Xue, Tao Zheng, J.R. Dahn, Carbon 34 (1996) 193-200.

[28] K. Tokumitsu, A. Mabuchi, H. Fujimoto, T. Kasuh, J. Electrochem. Soc. 143 (1996) 2235-2239.

[29] Kazuma Gotoh, Mariko Maeda, Aisaku Nagai, Atsushi Goto, Masataka Tansho, Kenjiro Hashi, Tadashi Shimizu, Hiroyuki Ishida, Journal of Power Sources 162 (2006) 1322-1328.

[30] Y.S. Yoon, S.H. Jee, S.H. Lee, S.C. Nam, Surface and Coatings Technology 206 (2011) $553-558$.

[31] Mingru Su, Zhixing Wang, Huajun Guo, Xinhai Li, Silin Huang, Wei Xiao, Lei Gan, Electrochimica Acta 116 (2014) 230-236.

[32] Mingru Su, Zhixing Wang, Huajun Guo, Xinhai Li, Silin Huang, Lei Gan, Advanced Powder Technology 24 (2013) 921-925.

[33] P. Limthongkul, Y.I. Jang, N.J. Dudney, Y.M. Chiang, Acta Materialia 51 (2003) 1103-1113.

[34] Yong-Mook Kang, Sang-Min Lee, Sang-Jin Kim, Goo-Jin Jeong, Min-Seok Sung, Wan-Uk Choi, Sung-Soo Kim, Electrochemistry Communications 9 (2007) 959-964.

[35] M. Obrovac and L. Christensen, Electrochemical and Solid State Lett., Vol. 7, Issue 5, p. A93 (2004) 